\title{
SELETIVIDADE DE PRODUTOS FITOSSANITÁRIOS UTILIZADOS NA CULTURA DA SOJA PARA PUPAS DE TRICHOGRAMMA PRETIOSUM RILEY, 1879 (HYMENOPTERA: TRICHOGRAMMATIDAE)
}

\author{
E.L. do Carmo ${ }^{1}$, A.F. Bueno ${ }^{2}$, R.C.O.F. Bueno ${ }^{1 *}$, S.S. Vieira ${ }^{3}$, M.M.P. Goulart ${ }^{1}$, T.R. Carneiro ${ }^{4}$
}

${ }^{1}$ Universidade de Rio Verde, CP 104, CEP 75901-970, Rio Verde, GO, Brasil. E-mail: adeney@cnpso.embrapa.br

\begin{abstract}
RESUMO
Oobjetivo do trabalho foi avaliar o efeito de produtos fitossanitários sobre pupas de Trichogramma pretiosum. Foram conduzidos três bioensaios em delineamento inteiramente casualizado com 11 tratamentos e cinco repetições. Em cada repetição, ovos de Anagasta kuehniella (Zeller, 1879) (Lepidoptera: Pyralidae) foram utilizados como hospedeiro alternativo, sendo aderidos em cartelas de cartolina de $1 \mathrm{~cm}^{2}$ com solução de goma arábica à $40 \%$ e oferecidos para o parasitismo por $24 \mathrm{~h}$. Após 168-192h do parasitismo, quando os parasitoides estavam na fase de pupa, as cartelas foram mergulhadas por cinco segundos nos tratamentos testados. Avaliou-se a sobrevivência dos indivíduos tratados e a redução na emergência dos parasitoides foi classificada segundoas normas da IOBC. Os inseticidas flufenoxurom (Cascade ${ }^{\circledR}$ ) 10, diflubenzurom (Dimilin ${ }^{\circledR}$ ) 20 e metoxifenozide (Intrepid $\left.240 \mathrm{SC}^{\circledR}\right) 21,6$ e 36 g i.a. ha-1 foram classificados como inócuos. Permetrina (Valon $384 \mathrm{CE}^{\circledR}$ ) 49,92; betaciflutrina 12,5 + imidacloprido 100 (Connect ${ }^{\circledR}$ ) e gama-cialotrina (Stallion $150 \mathrm{SC}^{\circledR}$ ) 3,75 gi.a. ha $^{-1}$ foram levemente nocivos. Clorpirifós (Lorban 480BR ${ }^{\circledR} 384$ e espinosade $\left(\operatorname{Tracer}^{\circledR}\right) 24$ g i.a. ha ${ }^{-1}$ foram moderadamente nocivos. Os herbicidas glifosato 720 + imazetapir 90 (Alteza ${ }^{\circledR}$ ); s-metolacloro (Dual

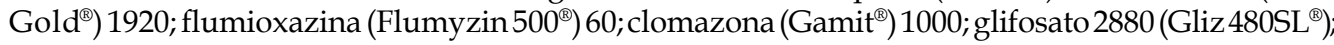
dicloreto de paraquate $600+$ diurom 300 (Gramocil $\left.{ }^{\circledR}\right)$; glifosato 1200 (Roundup Ready $\left.{ }^{\circledR}\right)$; foram classificados como inócuos e os herbicidas 2,4-D (DMA 806BR ${ }^{\circledR}$ ) 1209; dicloreto de paraquate ${\left(\text { Gramoxone }^{\circledR} \text { ) 600; glifosato (Roundup Transorb }\right.}^{\circledR}$ ) 2592 g i.a. ha ${ }^{-1}$ classificados como levemente nocivos. Os fungicidas tiofanato-metílico (Cercobin $500 \mathrm{SC}^{\circledR}$ ) 400; flutriafol 60 + tiofanato-metílico 300 (Celeiro $^{\circledR}$ ); carbendazin (Derosal500SC $^{\circledR}$ ) 250; tebuconazole $\left(\right.$ FolicurEC $\left.^{\circledR}\right)$ 150; flutriafol (Impact $\left.{ }^{\circledR}\right)$ 125; tebuconazole 120 + trifloxistrobina $60\left(\right.$ Nativo $\left.^{\circledR}\right)$; epoxiconazole 30 + piraclostrobina 79,8 $\left(\right.$ Opera $\left.^{\circledR}\right)$; epoxiconazole $\left(\right.$ OpusSC $\left.{ }^{\circledR}\right) 12,5$; azoxistrobina $\left(\right.$ Priori $\left.^{\circledR}\right) 50$; azoxistrobina $60+$ ciproconazole 24 (Priori Xtra ${ }^{\circledR}$ ) gi.a. ha ${ }^{-1}$ foram classificados como inócuos a T. pretiosumna fase de desenvolvimento avaliada (pupa). Dentre os produtos avaliados, aqueles classificados como inócuos devem ser priorizados no manejo integrado de pragas (MIP) por compatibilizar o uso do controle químico sem prejudicar o controle biológico. Do mesmo modo, os produtos classificados como moderadamente nocivos devem ser evitados sempre que possível ou substituídos por outro de menor impacto.
\end{abstract}

PALAVRAS-CHAVE: Controle biológico, controle químico, parasitoides de ovos, manejo integrado de pragas.

\begin{abstract}
SELECTIVITY OF PESTICIDES USED INSOYBEAN CROPSTOTRICHOGRAMMAPRETIOSUM RILEY, 1879 (HYMENOPTERA: TRICHOGRAMMATIDAE) PUPAE. This study aimed to evaluate the effect of different pesticides on Trichogrammapretiosumpupae. Three tests were carried out with 11 treatments and 5 replications. Eggs of Anagastakuehniella (Zeller, 1879) (Lepidoptera: Pyralidae) were used as a factitious host affixed on $1 \mathrm{~cm}^{2}$ pieces of cardboard using a non-noxious glue solution (40\% gum arabic) and then offered to parasitism for $24 \mathrm{~h}$. After 168-192 $\mathrm{h}$ from the parasitism, when the parasitoids were at the pupae stage, the pieces of cardboard were dipped for five seconds in the treatments. The parasitoid survival was evaluated and the emergence was classified accordingly to the IOBC guidelines. The insecticides flufenoxuron $\left(\right.$ Cascade $^{\circledR}$ ) 10, diflubenzuron (Dimilin $\left.{ }^{\circledR}\right) 20$
\end{abstract}

\footnotetext{
${ }^{2}$ Embrapa Soja, Londrina, PR, Brasil.

${ }^{3}$ Universidade do Estado de Santa Catarina, Florianópolis, SC, Brasil.

${ }^{4}$ Universidade Estadual Paulista, Faculdade de Ciências Agrárias e Veterinárias, Jaboticabal, SP, Brasil.

*Bolsista Pós-Doutorado CAPES/PNPD - FESURV.
} 
and methoxyfenozide (Intrepid 240SC ${ }^{\circledR}$ ) 21.6 and $36 \mathrm{~g}$ a.i. ha ${ }^{-1}$ were classified as harmless. Permethrin (Valon 384CE ${ }^{\circledR}$ ) 49.92, beta-cyfluthrin $12.5+$ imidacloprid 100 (Connect $^{\circledR}$ ) and gammacyhalothrin (Stallion $\left.150 \mathrm{SC}^{\circledR}\right) 3.75 \mathrm{~g}$ a.i. ha ${ }^{-1}$ were slightly harmful. Chlorpyriphos (Lorsban 480BR $\left.{ }^{\circledR}\right) 384$ and spinosad $\left(\right.$ Tracer $\left.^{\circledR}\right) 24 \mathrm{~g}$ a.i. ha ${ }^{-1}$ were moderately harmful. The herbicides glyphosate $720+$ imazetapyr $90\left(\right.$ Alteza $^{\circledR}$ ), s-metolachlor $\left(\right.$ DualGold $\left.^{\circledR}\right) 1920$, flumyoxazin (Flumyzin $\left.500^{\circledR}\right)$ 60, clomazone (Gamite ${ }^{\circledR}$ ) 1000, glyphosate $\left(\mathrm{Gliz}^{\circledR}\right)$ 2880, paraquat dichloride 600 + diuron 300 $\left(\right.$ Gramocil $\left.^{\circledR}\right)$, glyphosate 1200 (Roundup Ready ${ }^{\circledR}$ ) were classified as harmless, and the herbicides 2.4-D (DMA ${ }^{\circledR}$ ) 1209, paraquat dichloride (Gramoxone ${ }^{\circledR}$ ) 600 and glyphosate (Roundup Transorb ${ }^{\circledR}$ ) $2592 \mathrm{~g}$ a.i. ha ${ }^{-1}$ wereclassified as slightly harmful. The fungicides metil tiofanate (Cercobin $500 \mathrm{SC}{ }^{\circledR}$ ) 400, flutriafol $60+$ metil tiofanate $300\left(\right.$ Celeiro $^{\circledR}$ ), carbendazin (Derosal 500SC ${ }^{\circledR}$ ) 250, tebuconazol (Folicur EC ${ }^{\circledR}$ ) 150, flutriafol $\left(\right.$ Impact $\left.^{\circledR}\right)$ 125, tebuconazol $120+$ trifloxystrobin $60\left(\mathrm{Nativo}^{\circledR}\right)$, epoxiconazol $30+$ piraclostrobin 79.8 (Opera ${ }^{\circledR}$ ), epoxiconazol (Opus SC ${ }^{\circledR}$ ) 12.5, azoxystrobin (Priori $^{\circledR}$ ) 50, azoxystrobin $60+$ ciproconazol 24 (Priori Xtra $^{\circledR}$ ) ga.i. ha ${ }^{-1}$ were harmless to T.pretiosum pupae. Among the tested products, the harmless ones should be chosen in a program of integrated pest management (IPM) since it allows the use of pesticides without harm to the biological control. On the other hand, the moderately harmful pesticides should be avoided or replaced by another product with less impact whenever it is possible.

KEY WORDS: Biological control, chemical control, egg parasitoids, integrated pest management

\section{INTRODUÇÃO}

A cultura da soja é uma das principais atividades geradoras de divisas ao país tendo grande participaçãona economia nacional. De acordocom levantamento realizado no mês de março pelo IBGE (2008), a área cultivada com o grão foi de, aproximadamente, 21,11 milhões de hectares, resultando em uma produção estimada de 59,9 milhões de toneladas. Mesmo com essa área cultivada, o aumento da demanda por produtos alimentícios faz com que novas áreas de cultivo sejam abertas e exploradas para a produção agrícola. Essefato, aliadoà baixa tecnologia dealgumas regiões, leva a um aumento abusivo do uso de produtos fitossanitários, causando a contaminação do ambiente. Uma parcela significativa do desequilíbrio biológico que ocorre em sistemas agrícolas deve-se ao uso inadequado desses produtos. Aplicados de maneira indiscriminada, os agrotóxicos podem levar à ressurgência, surtos de pragas secundárias e seleção de insetos resistentes (Diez-Rodríguez; Омото, 2001).

Uma alternativa para a sustentabilidade do agronegócio éo uso de táticas de manejo integrado de pragas (MIP) que visem compatibilizar o uso do controle biológico com inseticidas seletivos aos inimigos naturais. A seletividadeé a chave do MIPem sistemas que visam reduzir a população de insetos nocivos, sem alterar ou impactar o mínimo possível os outros componentes do agroecossistema e do ambiente(SANTos et al., 2006).

Os produtos químicos só podem ser associados a outras táticas de manejo adotadas em MIP seapresentar algum grau de seletividade (SANTOS et al., 2006) e, por isto, o controle biológico merece destaque devido à sua sustentabilidade e baixo risco ao homem e ao ambiente. Entre os diversos agentes de controle biológico, os parasitoides de ovos do gênero Trichogramma têm sido muito usados na agricultura, principalmente devido à sua facilidade de criação e agressividade no controle das pragas (PARRA; ZUCCHI, 2004).

Estudos com parasitoides do gêneroTrichogramma vêm sendo conduzidos em mais de 50 países e liberações realizadas comercialmente todo ano em mais de 32 milhões de hectares (BuENo et al., 2009). No Brasil, a utilização de Trichogramma pretiosum Riley, 1879 (Hymenoptera: Trichogrammatidae) é ainda pequena se comparada com outros países (PARRA etal.,2002). Alguns estudos com esse parasitoide têm sido realizados visando ao controle biológico de pragas da cana-de-açúcar (BотеLHo et al., 1999), do algodoeiro (Bleicher; Parra, 1989), do tomateiro (Pratissoli;P arra, 2000), do milho (SÁ;PARRA, 1994), de pragas de grãos armazenados (INOUE; PARRA, 1998), citros (MATOS, 2007), dentre outros. O uso dos parasitoides Trichogramma spp. no controle de lepidópteros que atacam a cultura da soja tem grande potencial de sucesso, visto que esses inimigos naturais parasitam ovos de praticamente todo complexo de pragas da ordem Lepidoptera que ataca a cultura (BUENo et al., 2007). Entretanto, o uso do controle químico é ainda indispensável no sistema de cultivo atual, sendo o método de controle mais utilizados pelos produtores agrícolas. Sendo assim, a finalidade do presente trabalho foi avaliar a seletividade de produtos fitossanitários normalmente utilizados na cultura da soja sobre pupas de T.pretiosum visando identificar aqueles que causem menor impacto sobre esse inimigo natural para que sejam os produtos priorizados para uso quando necessário.

\section{MATERIAL E MÉTODOS}

Foram conduzidos três bioensaios em condições controladas de laboratório $\left(25 \pm 2^{\circ} \mathrm{C}\right.$, UR de $70 \pm 10 \%$ 
e fotofase de 12 horas) em delineamento inteiramente casualizado com 11 tratamentos (Tabelas 1, 2 e 3 ) e cinco repetições, conforme metodologia deBuENo etal. (2008) e CARMoet al.(2009), sendo que a população de T. pretiosum foi multiplicada em ovos de A. kuehniella, considerado o melhor hospedeiro alternativo para a criação dessa espécie de Trichogramma (PARRA;Z UCCHI, 2004).

Para cada repetição foi utilizados uma cartela de cartolina branca de $1 \mathrm{~cm}^{2}$ com cerca de 200 ovos de $A$. kuehniella contendo o parasitoide na fase de pupa (168-192 horas após o parasitismo) (Consoli et al., 1999). Essas cartelas contendo os ovos parasitados foram identificadas e depois imersas por cinco segundos nas caldas químicas, que foram preparadas considerando-se volume de aplicação de $200 \mathrm{~L} \mathrm{ha}^{-1}$. Após secagem sob papel absorvente por duas horas, as cartelas foram introduzidas em sacos plásticos transparentes ( $4 \mathrm{~cm}$ de largura $\times 15 \mathrm{~cm}$ de comprimento) e acondicionadas em ambiente controlado $\left(25 \pm 2^{\circ} \mathrm{C}\right.$, UR de $70 \pm 10 \%$ efotofase de 12 horas) até a emergência dos adultos.

A viabilidade do parasitismo (\%) foi avaliada contando-se os ovos parasitados e os que efetivamente tiveram a emergência dos parasitoides. A redução na emergência de T.pretiosum em relação ao tratamento testemunha foi calculada pela seguinte equação: $\mathrm{E}$ $(\%)=\left(1-V_{t} / V_{c}\right) \times 100$, onde: $E(\%)$ é a porcentagem de redução da viabilidade do parasitismo; Vté a viabilidade do parasitismo médio para o tratamento testado e Vc é a viabilidade do parasitismo médio observado para o tratamento testemunha (MANZONI et al., 2007).

Tabela 1 - Inseticidas testados (bioensaio 1) sobre pupas de Trichogramma pretiosum em condições controladas de laboratório (AGROFIT, 2008).

\begin{tabular}{|c|c|c|c|c|c|}
\hline Inseticida (p.c.) & Formulação & Igrediente ativo (i.a.) & Grupo Químico & (g) i.a./ $200 \mathrm{~L} \mathrm{H}_{2} \mathrm{O}$ & p.c./ $200 \mathrm{~L} \mathrm{H}_{2} \mathrm{O}$ \\
\hline Cascade & $100 \mathrm{CE}$ & Flufenoxurom & Benzoilureia & 10 & $0,1 \mathrm{~L}$ \\
\hline Connect & 100/12.5 SC & $\begin{array}{l}\text { Imidacloprido + } \\
\text { beta-ciflutrina }\end{array}$ & $\begin{array}{l}\text { Neonicotinoide }+ \\
\text { Piretroide }\end{array}$ & $100+12.5$ & $0,1 \mathrm{~L}$ \\
\hline Dimilin & $80 \mathrm{WG}$ & Diflubenzurom & Benzoilureia & 20 & $0,08 \mathrm{~kg}$ \\
\hline Intrepid & $24 \mathrm{SC}$ & Metoxifenozide & Diacilhidrazina & 21,6 & $0,09 \mathrm{~L}$ \\
\hline Intrepid & $24 \mathrm{SC}$ & Metoxifenozide & Diacilhidrazina & 36 & $0,15 \mathrm{~L}$ \\
\hline Lorsban & $480 \mathrm{CE}$ & Clorpirifós & Organofosforado & 384 & $0,8 \mathrm{~L}$ \\
\hline Stallion & $150 \mathrm{CS}$ & Gama-cialotrina & Piretroide & 3,75 & $0,025 \mathrm{~L}$ \\
\hline Talstar & $100 \mathrm{EC}$ & Bifentrina & Piretroide & 5 & $0,05 \mathrm{~L}$ \\
\hline Tracer & 480 SC & Espinosade & Espinosina & 24 & $0,05 \mathrm{~L}$ \\
\hline Valon & $384 \mathrm{CE}$ & Permetrina & Piretroide & 49,92 & $0,13 \mathrm{~L}$ \\
\hline
\end{tabular}

Tabela 2 - Herbicidas testados (bioensaio 2) sobre pupas de Trichogramma pretiosum em condições controladas de laboratório (AGROFIT, 2008).

\begin{tabular}{|c|c|c|c|c|c|}
\hline Herbicida (p.c.) & Formulação & Igrediente ativo (i.a.) & Grupo Químico & (g) i.a./ $200 \mathrm{~L} \mathrm{H}_{2} \mathrm{O}$ & p.c./ $200 \mathrm{~L} \mathrm{H}_{2} \mathrm{O}$ \\
\hline Alteza & $240 / 30 \mathrm{SL}$ & $\begin{array}{l}\text { Glifosato + } \\
\text { imazetapir }\end{array}$ & $\begin{array}{l}\text { Imidazolinona }+ \\
\text { glicina substituída }\end{array}$ & $720+90$ & $3 \mathrm{~L}$ \\
\hline DMA & $806 \mathrm{SL}$ & 2.4-D & $\begin{array}{l}\text { Ácido } \\
\text { ariloxialcanóico }\end{array}$ & 1209 & $1,5 \mathrm{~L}$ \\
\hline Dual Gold & $960 \mathrm{EC}$ & S-metolacloro & $\begin{array}{l}\text { Cloroacetanilida } \\
\text { ciclohexenodicarb }\end{array}$ & 1920 & $2 \mathrm{~L}$ \\
\hline Flumyzin & $500 \mathrm{WP}$ & Flumioxazina & oximida & 60 & $0,12 \mathrm{~kg}$ \\
\hline Gamit & $500 \mathrm{EC}$ & Clomazona & Isoxazolidinone & 1000 & $2 \mathrm{~L}$ \\
\hline Gliz & $480 \mathrm{SL}$ & Glifosato & Glicina substituída & 2880 & $6 \mathrm{~L}$ \\
\hline Gramocil & $100 / 200$ SC & $\begin{array}{l}\text { Diuron }+ \text { dicloreto } \\
\text { de paraquate }\end{array}$ & Uréia + bipiridílio & $300+600$ & $3 \mathrm{~L}$ \\
\hline Gramoxone & 200 SL & $\begin{array}{l}\text { dicloreto de } \\
\text { paraquate }\end{array}$ & Bipiridílio & 600 & $3 \mathrm{~L}$ \\
\hline Roundup Ready & $480 \mathrm{SL}$ & Glifosato & Glicina substituída & 1200 & $2,5 \mathrm{~L}$ \\
\hline Roundup Transorb & $648 \mathrm{SL}$ & Glifosato & Glicina substituída & 2592 & $4 \mathrm{~L}$ \\
\hline
\end{tabular}


Tabela 3 - Fungicidas testados (bioensaio 3) sobre pupas de Trichogramma pretiosum em condições controladas de laboratório (AGROFIT, 2008).

\begin{tabular}{|c|c|c|c|c|c|}
\hline Fungicida (p.c.) & Formulação & Igrediente ativo (i.a.) & Grupo Químico & (g) i.a./ $200 \mathrm{~L} \mathrm{H}_{2} \mathrm{O}$ & p.c./ $200 \mathrm{~L} \mathrm{H}_{2} \mathrm{O}$ \\
\hline Celeiro & $100 / 500 \mathrm{SC}$ & $\begin{array}{l}\text { Flutriafol + } \\
\text { Tiofanato metílico }\end{array}$ & $\begin{array}{l}\text { Triazol + } \\
\text { benzimidazol }\end{array}$ & $60+300$ & $0,6 \mathrm{~L}$ \\
\hline Cercobim & 500 SC & Tiofanato metílico & Benzimidazol & 400 & $0,8 \mathrm{~L}$ \\
\hline Derosal & $500 \mathrm{SC}$ & Carbendazin & Benzimidazol & 250 & $0,5 \mathrm{~L}$ \\
\hline Folicur & $250 \mathrm{EC}$ & Tebuconazole & Triazol & 150 & $0,75 \mathrm{~L}$ \\
\hline Impact & $125 \mathrm{SC}$ & Flutriafol & Triazol & 125 & $1 \mathrm{~L}$ \\
\hline Nativo & $100 / 200$ SC & $\begin{array}{l}\text { Trifloxistrobina }+ \\
\text { Tebuconazole }\end{array}$ & $\begin{array}{l}\text { Estrobilurina + } \\
\text { triazol }\end{array}$ & $60+120$ & $0,6 \mathrm{~L}$ \\
\hline Opera & $50 / 133 \mathrm{SE}$ & $\begin{array}{l}\text { Epoxiconazole + } \\
\text { Piraclostrobina }\end{array}$ & $\begin{array}{l}\text { Triazol + } \\
\text { estrobilurina }\end{array}$ & $30+79.8$ & $0,6 \mathrm{~L}$ \\
\hline Opus & $125 \mathrm{SC}$ & Epoxiconazole & Triazol & 12,5 & $0,1 \mathrm{~L}$ \\
\hline Priori & $250 \mathrm{SC}$ & Azoxistrobina & Estrobilurina & 50 & $0,2 \mathrm{~L}$ \\
\hline Priori Xtra & $200 / 80$ SC & $\begin{array}{l}\text { Azoxistrobina }+ \\
\text { Ciproconazole }\end{array}$ & $\begin{array}{l}\text { Estrobilurina + } \\
\text { triazol }\end{array}$ & $60+24$ & $0,3 \mathrm{~L}$ \\
\hline
\end{tabular}

Em função das médias de redução, os produtos foram enquadrados de acordo com as normas preconizadas pela "International Organization of Biological Control of Noxious Animals and Plants" (IOBC), em: classe 1 - inócuo ( $\mathrm{E}<30 \%$ ); classe 2 levemente nocivo $(30 \leq \mathrm{E} \leq 79 \%)$; classe 3 - moderadamente nocivo ( $80 \leq \mathrm{E} \leq 99 \%)$; classe 4 - nocivo ( $\mathrm{E}>99 \%$ ) (HASSAN, 1992).

Os resultados obtidos de viabilidade do parasitismo (\%) foram submetidos às análises exploratórias para avaliar as pressuposições de normalidade dos resíduos, homogeneidade de variância dos tratamentos, aditividade do modelo para permitir a aplicação da ANOVA (SAS INSTituTe, 2001). As médias foram comparadas pelo teste deScott-Knott a $5 \%$ de significância utilizando-se o programa estatístico SISVAR.

\section{RESULTADOS E DISCUSSÃO}

Flufenoxurom 10, diflubenzurom 20 e metoxifenozide 21,6 e 36 g i.a. ha- ${ }^{-1}$ apresentaram viabilidade do parasitismo semelhante à observada no tratamento testemunha e foram serem classificados como inócuos (classe 1) a pupa de T. pretiosum (Tabela 4).

Flufenoxurom e diflubenzurom são inseticidas do grupo das benzoilfenilureias, classificados como reguladores de crescimento que inibem a síntese de quitina, sendo venenos estomacais de ação lenta (REYNOLDS, 1987). Metoxifenozide é um inseticida do grupo das diacilhidrazinas, sendo também um regulador de crescimento, mas que atua como agonista de ecdisteroide, provocando a aceleração do processo de ecdise, porém seu exato modo de ação ainda é desconhecido (DHADiallaet al., 1998).

Os inseticidas reguladores de crescimento são normalmente destacados pela sua seletividade aos agentes de controle biológico (SANTOS et al. 2006). Entretanto, em trabalhos realizados por BUENO;FREITAS (2004) e BuENo et al. (2008), observou-se que alguns desses inseticidas atuaram de forma não seletiva a Crysoperla externa (Hagen, 1861) (Neuroptera: Chrysopidae) e T.pretiosum, respectivamente. Deacordo com Pratissoliet al. (2004), lufenuron, um inseticida do grupo dos reguladores de crescimento, ocasionou redução no parasitismo por T. pretiosum, quando este foi comparado ao tratamento testemunha. Entretanto, é importante salientar quePraTissoliet al.(2004) avaliaram a fase de adulto enquanto esse trabalho refere-se à fase de pupa do parasitoide que, por estar protegida dentro do córion do ovo hospedeiro, é sempre considerada como a fase mais resistente dos parasitoides de ovos à ação dos agrotóxicos (HASSAN, 1992; SANTOS et al., 2006). Isto mostra a importância de realizar esses estudos com os diferentes produtos fitossanitários, além das diferentes espécies efases de desenvolvimento dos inimigos naturais.

Bifentrina foi o único inseticida fora do grupo dos regulares de crescimento de insetos que foi classificado como inócuo (classe 1) na dose estudada a pupas de T. pretiosum, apesar da viabilidade do parasitismo ser estatisticamente inferior a testemunha (Tabela 4). Esteinseticidaéum produto do grupo dos piretroides, que são inseticidas neurotóxicos normalmente considerados de baixa seletividade aos inimigos naturais (CAÑETE, 2005). Beta-ciflutrina, permetrina e gamacialotrina foram os demais inseticidas avaliados do grupo dos piretroides sendo classificados como levemente nocivo (classe 2), sendo que a viabilidade do 
parasitismo observado nesses tratamentos foram todas inferiores a testemunha (Tabela 4). O reduzido impacto dos piretroides a pupas deT. pretiosum observados nesse experimento deve-se provavelmente, como já mencionado anteriormente, pelo fato da fase de pupa estar protegida dentro do córion do ovo hospedeiro, o que a deixa mais resistente a ação dos inseticidas. Oefeito esperado desses produtos na fase adulta de $T$. pretiosum, que é mais sensível aos agrotóxicos, é possivelmente diferente dos resultados obtidos para pupa. Ainda, CARvalHo et al. (1999) observaram que o piretroide lambdacialotrina $(0,025$ g i.a./L. de água) provocou reduzida capacidade de parasitismo de adultos de duas linhagens de $T$. pretiosum por até 31 dias devido à ação desse inseticida, efeito subletal da ação dos inseticidas que não foi avaliado neste experimento.

Clorpirifós 384 gi.a. ha-1 reduziu a viabilidade do parasitismo, sendo enquadrado na classe 3 (moderamente nocivo). BuENo et al. (2008) relataram classificação mais seletiva para esse produto na dosagem de $240 \mathrm{~g} / 200 \mathrm{~L}$ de água para as fase de ovo, larva e pupa de T. pretiosum. Entretanto, isto se deve provavelmente à diferença de dosagem utilizada na condução dos boiensaios. A toxicidadeou seletividade de um agrotóxico é sempre dependente da dosagem avaliada (SANTOS etal., 2006), o que mostra a importância de se concluir e comparar resultados de seletividade sempre levando em consideração a dose dos produtos em estudo.

Espinosade $24 \mathrm{~g}$ i.a. ha-1 foi classificado como moderadamente nocivo (classe 3 ) por promover redu- ção na emergência dos parasitoides de $82,24 \%$ em relação ao tratamento testemunha. Resultados semelhantes com esse produto na dose de $12 \mathrm{~g}$ i.a. ha- ${ }^{-1}$ foram obtidos porCAÑETE (2005) para cinco diferentes espécies de Trichogramma. O espinosade é um inseticida de origem natural, oriundo da fermentação da bactéria Sacharopolyspora spinosa edescrito como seletivo a alguns inimigos naturais como predadores, mas normalmente mais nocivo para parasitoides da ordem Hymenoptera (Williams et al., 2003). Entretanto, a orgânica, comercializada nos EUA, é diferente da formulação testada no presente trabalho. Diferenças entreinertes podem ser responsáveis pela seletividade ou não de um agrotóxico aos inimigos naturais (NöRNBERG et al., 2008) e podem ser responsáveis por algumas das diferenças encontradas na literatura. Resultados nocivos do espinosade foram também relatados por BUENO et al. (2008) utilizando a mesma formulação utilizada nesse bioensaio em todas as fases imaturas de T. pretiosum.

Beta-ciflutrina 12,5 + imidacloprido $100 \mathrm{~g}$ i.a. ha ${ }^{-1}$ foi classificado como levemente nocivo (classe 2) à fase de pupa de T. pretiosum. Resultados semelhantes foram obtidos por MATOs (2007) que não observou efeito nocivo do imidacloprido sobre a fase de pupa do T. pretiosum. A mesma dosagem doagrotóxico quando aplicado em pupas de Telenomus remus Nixon, 1937 (Hymenotpera: Scelionidae) foi classificado como seletivo (CARMoetal., 2009) que se mostrou ainda mais tolerante a ação desse inseticida. Sendo assim, esse produto se mostrou compatível para uso no MIP por preservar a ação do parasitoide T. pretiosum.

Tabela 4 - Efeito (E) de inseticidas na viabilidade do parasitismo de Trichogramma pretiosum após a imersão de ovos de Anagasta kuehniella contendo pupas do parasitoide por cinco segundos em diferentes compostos diluídos em 200 L de água.

\begin{tabular}{|c|c|c|c|}
\hline Tratamento (g i.a. ha ${ }^{-1}$ ) & Viabilidade $(\%)^{1}$ & $\mathrm{E}(\%)^{2}$ & Classe $^{3}$ \\
\hline Permetrina 49,92 & $24,30 \pm 6,13 c$ & 61,57 & 2 \\
\hline Flufenoxurom 10 & $60,24 \pm 3,47 \mathrm{a}$ & 4,75 & 1 \\
\hline Beta-ciflutrina 12,5 + imidacloprido100 & $36,75 \pm 3,69 b$ & 42,72 & 2 \\
\hline Diflubenzurom 20 & $65,76 \pm 2,56 \mathrm{a}$ & 0,00 & 1 \\
\hline Bifentrina 5 & $47,36 \pm 4,35 \mathrm{~b}$ & 25,12 & 1 \\
\hline Metoxifenozide 21,6 & $57,31 \pm 4,12 \mathrm{a}$ & 9,38 & 1 \\
\hline Metoxifenozide 36 & $60,68 \pm 4,12 \mathrm{a}$ & 4,05 & 1 \\
\hline Clorpirifós 384 & $0,69 \pm 0,04 \mathrm{~d}$ & 98,91 & 3 \\
\hline Gama-cialotrina 3,75 & $37,64 \pm 6,05 b$ & 40,48 & 2 \\
\hline Espinosade 24 & $11,23 \pm 1,30 \mathrm{~d}$ & 82,24 & 3 \\
\hline Água (testemunha) & $63,24 \pm 1,89 a$ & - & - \\
\hline$C V(\%)=35,34$ & & - & - \\
\hline
\end{tabular}


Os herbicidas glifosato 720 + imazetapir 90, smetolacloro 1920, flumioxazina 60, clomazona 1000, glifosato 2880, dicloreto de paraquate $600+$ diurom 300 e glifosato 1200 foram classificados como inócuos (classe 1) e os herbicidas 2,4-D 1209, dicloreto de paraquate 600 e glifosato $2592 \mathrm{~g}$ i.a. ha- ${ }^{-1}$ classificados como levemente nocivos (classe 2) (Tabela 5). Resultados similares aos observados no presente trabalho foram relatados por BUENOet al.(2008) paraoclorimuron, lactofen, fomesafen, fluzifop e diferentes dosagens de glifosato sobre larvas e pupas de T. pretiosum. Semelhante aos herbicidas, os fungicidas testados foram classificados como inócuos (classe 1) (Tabela 6). Esses resultadosindicam quea aplicação deherbicidas ou de fungicidas não tem grande impacto na população de T. pretiosum, quando este se encontra em pupa.

Tabela 5 - Efeito (E) de herbicidas na viabilidade do parasitismo de Trichogramma pretiosum após a imersão de ovos de Anagasta kuehniella contendo pupas do parasitoide por cinco segundos em diferentes compostos diluídos em 200 L de água.

\begin{tabular}{|c|c|c|c|}
\hline Tratamento (g i.a. ha ${ }^{-1}$ ) & Viabilidade $(\%)^{1}$ & $\mathrm{E}(\%)^{2}$ & Classe $^{3}$ \\
\hline Glifosato 720 + imazetapir 90 & $44,91 \pm 2,67 \mathrm{bcd}$ & 27,12 & 1 \\
\hline 2,4-D 1209 & $38,63 \pm 1,57 \mathrm{~cd}$ & 37,31 & 2 \\
\hline S-metolacloro 1920 & $58,94 \pm 4,36$ a & 4,36 & 1 \\
\hline Flumioxazina 60 & $51,30 \pm 3,15 \mathrm{ab}$ & 16,76 & 1 \\
\hline Clomazona 1000 & $50,60 \pm 3,19 a b c$ & 17,89 & 1 \\
\hline Glifosato $2880\left(\mathrm{Gliz}^{\circledR}\right)$ & $51,69 \pm 1,98 \mathrm{ab}$ & 16,13 & 1 \\
\hline Dicloreto de paraquate $600+$ diurom 300 & $45,05 \pm 0,58 \mathrm{bcd}$ & 26,90 & 1 \\
\hline Dicloreto de paraquate 600 & $36,19 \pm 3,87 d$ & 41,28 & 2 \\
\hline Glifosato 1200 (Roundup Ready ${ }^{\circledR}$ ) & $50,83 \pm 1,01 \mathrm{ab}$ & 17,51 & 1 \\
\hline Glifosato 2592 (Roundup Transorb ${ }^{\circledR}$ ) & $41,61 \pm 2,59 \mathrm{bcd}$ & 32,48 & 2 \\
\hline Água (testemunha) & $61,63 \pm 1,20 \mathrm{a}$ & - & - \\
\hline
\end{tabular}

${ }^{1}$ Médias seguidas pela mesma letra na coluna não diferem estatisticamente entre si pelo teste de Scott-Knott $(\mathrm{P}<0,05)$; ${ }^{2} \mathrm{E}(\%)=(1-\mathrm{Vt} / \mathrm{Vc}) \times 100$ (MANZONi et al., 2007);

${ }^{3}$ classe 1 - inócuo $(\mathrm{E}<30 \%)$, classe 2 - levemente nocivo ( $\left.30 \leq \mathrm{E} \leq 79 \%\right)$, classe 3 - moderadamente nocivo $(80 \leq \mathrm{E} \leq 99 \%)$ e classe 4 - nocivo (E > 99\%) (Hassan, 1992).

Tabela 6 - Efeito (E) de fungicidas na viabilidade do parasitismo de Trichogramma pretiosumapós a imersão de ovos de Anagasta kuehniella contendo pupas do parasitoide por cinco segundos em diferentes compostos diluídos em $200 \mathrm{~L}$ de água.

\begin{tabular}{lcrc}
\hline Tratamento (g i.a. ha ${ }^{-1}$ ) & Viabilidade $(\%)^{1}$ & E (\%) ${ }^{2}$ & Classe $^{3}$ \\
\hline Tiofanato-metílico 400 & $45,63 \pm 1,45 \mathrm{ab}$ & 2,42 & 1 \\
Flutriafol 60 + tiofanato-metílico 300 & $40,92 \pm 2,69 \mathrm{ab}$ & 12,50 & 1 \\
Carbendazin 250 & $42,03 \pm 5,50 \mathrm{ab}$ & 10,13 & 1 \\
Tebuconazole 150 & $40,70 \pm 2,87 \mathrm{~b}$ & 12,96 & 1 \\
Flutriafol 125 & $52,14 \pm 2,25 \mathrm{ab}$ & 0,00 & 1 \\
Tebuconazole120 + trifloxistrobina 60 & $55,37 \pm 1,99 \mathrm{a}$ & 0,00 & 1 \\
Epoxiconazole 30 + piraclostrobina79,8 & $53,31 \pm 1,66 \mathrm{ab}$ & 0,00 & 1 \\
Epoxiconazole 12,5 & $45,27 \pm 4,56 \mathrm{ab}$ & 0,00 & 1 \\
Azoxistrobina 50 & $49,88 \pm 3,22 \mathrm{ab}$ & 0,00 & 1 \\
Azoxistrobina 60 + ciproconazole 24 & $51,93 \pm 2,13 \mathrm{ab}$ & 0,00 & 1 \\
Água (testemunha) & $46,76 \pm 1,77 \mathrm{ab}$ & - \\
\hline CV (\%) = 13,79 & & - & -
\end{tabular}

${ }^{1}$ Médias seguidas pela mesma letra na coluna não diferem estatisticamente entre si pelo teste de Scott-Knott $(\mathrm{P}<0,05)$; ${ }^{2} \mathrm{E}(\%)=(1-\mathrm{Vt} / \mathrm{Vc}) \times 100$ (MANZONi et al., 2007);

${ }^{3}$ classe 1 - inócuo $(\mathrm{E}<30 \%)$, classe 2 - levemente nocivo $(30 \leq \mathrm{E} \leq 79 \%)$, classe 3 - moderadamente nocivo $(80 \leq \mathrm{E} \leq 99 \%)$ e classe 4 - nocivo (E $>99 \%)$ (HASSAN, 1992). 
É importante, ainda, salientar que, nas condições delaboratório, os ovos parasitados foram submetidos à máxima exposição aos produtos eque os compostos que se revelaram tóxicos para T. pretiosum podem ter seus efeitos reduzidos por vários fatores, quando aplicados em situação de campo. Um desses fatores é o fato de que a degradação dos compostos pela ação da luz geralmenteé acelerada nessa condição (RochA; CARvalHo, 2004). Dessa forma, recomenda-se que os produtos classificados como classe 2,3 e 4 em condições de laboratório sejam, também, testados em condições de semicampo e campo, conforme recomendado pelos protocolos da IOBC (HASSAN, 1992).

\section{CONCLUSÕES}

Os inseticidas flufenoxurom $\left(\right.$ Cascade $\left.^{\circledR}\right)$ 10, diflubenzurom (Dimilin ${ }^{\circledR}$ )20emetoxifenozide(Intrepid $\left.240 S C^{\circledR}\right)$ 21,6 e 36 g i.a. ha-1 ${ }^{-1}$ os herbicidas glifosato 720 + imazetapir 90 (Alteza ${ }^{\circledR}$ ), s-metolacloro (Dual Gold ${ }^{\circledR}$ ) 1920, flumioxazina (Flumyzin 500 ${ }^{\circledR}$ ) 60, clomazona

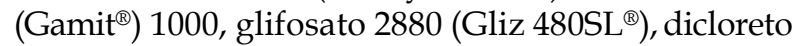

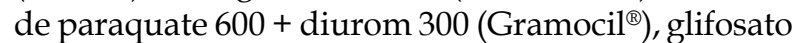
1200 (Roundup Ready ${ }^{\circledR}$ ) e os fungicidas tiofanatometílico(Cercobin500SC ${ }^{\circledR}$ ) 400, flutriafol60+ tiofanatometílico 300 (Celeiro ${ }^{\circledR}$ ), carbendazin (Derosal 500SC ${ }^{\circledR}$ ) 250, tebuconazole (FolicurEC ${ }^{\circledR}$ ) 150, flutriafol $\left(\right.$ Impact $^{\circledR}$ ) 125 , tebuconazole $120+$ trifloxistrobina 60 (Nativo ${ }^{\circledR}$ ), epoxiconazole $30+$ piraclostrobina 79,8 (Opera ${ }^{\circledR}$ ), epoxiconazole (Opus SC ${ }^{\circledR}$ ) 12,5 , azoxistrobina (Priori ${ }^{\circledR}$ )

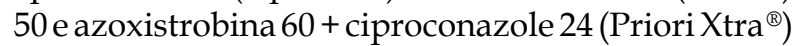
g i.a. ha-1 foram classificados como inócuos (classe 1) à pupa de T. pretiosum e seu uso é compatível com a preservação desse inimigo natural.

Os inseticidas permetrina (Valon $384 \mathrm{CE}^{\circledR}$ ) 49,92, beta-ciflutrina 12,5 + imidacloprido 100 (Connect $^{\circledR}$ ), gama-cialotrina (Stallion $150 \mathrm{SC}^{\circledR}$ ) 3,75 g i.a. ha-1 e os herbicidas 2,4-D (DMA 806BR ${ }^{\circledR}$ ) 1209, dicloreto de paraquate (Gramoxone ${ }^{\circledR}$ ) 600 e glifosato (Roundup Transorb $\left.^{\circledR}\right) 2592$ gi.a. ha ${ }^{-1}$ classificados comolevemente nocivos (classe 2) e quando possível devem ser substituídos por produtos cuja a classificação for inferior.

Por outro lado, os inseticidas clorpirifós (Lorban 480 BR $^{\circledR}$ ) 384 e espinosade $\left(\right.$ Tracer $^{\circledR}$ ) 24 g.i.a. ha ${ }^{-1}$ foram moderadamente nocivos (classe 3) e seu uso não é compatível com a preservação de T. pretiosum.

\section{AGRADECIMENTOS}

À Embrapa Arroz e Feijão e Embrapa Soja, CNPq e CAPES pelo apoio na realização da pesquisa. Aos pesquisadores da Embrapa Soja Dra. Lenita J. Oliveira (in memorian) e Dra. Maria Cristina Neves deOliveira pelas valiosas colaborações na revisão do manus- crito. Este artigo foi revisado e aprovado para publicação pelo Comitê de Publicações da Embrapa Soja sob o número 15/2008.

\section{REFERÊNCIAS}

AGROFIT. Sistema de agrotóxicos fitossanitários. Disponível em: <http:// www.extranet.agricultura.gov.br/ agrofit_cons/principal_agrofit_cons $>$. Acesso em: 28 abr. 2008.

BLEICHER, E.; PARRA, J.R.P. Espécies de Trichogramma parasitóides de Alabama argillacea. I. Biologia de três populações. Pesquisa Agropecuária Brasileira, v.24, p.929940, 1989.

BOTELHO, P.S.M.; PARRA, J.R.P.; CHAGAS NETO, J.F.; OLIVEIRA, C.P.B. Associação do parasitóide de ovos Trichogramma galloi Zucchi (Hymenoptera:

Trichogrammatidae) e do parasitóide larval Cotesia flavipes (Cam.) (Hymenoptera: Braconidae) no controle de Diatraea saccharalis (Fabr.) (Lepdoptera: Crambidae) em cana-de- açúcar. Anais da Sociedade Entomológica do Brasil, v.28, p.491-496, 1999.

BUENO, A.F.; FREITAS, S. Effect of the insecticides abamectin and lufenuron on eggs and larvae of Chrysoperla externa under laboratory conditions. BioControl, v.49, n.3, p.277-283, 2004.

BUENO, R.C.O.F.; PARRA, J.R.P.; BUENO, A.F.; MOSCARDI, F.; Di OLIVEIRA, J.R.G.; CAMILLO, M.F. Sem barreira. Cultivar, v.93, p.12-15, 2007.

BUENO, A.F.; BUENO, R.C.O.F.; PARRA, J.R.P.; VIEIRA, S.S. Effects of pesticides used in soybean crops to the egg parasitoid Trichogramma pretiosum. Ciência Rural, v.38, n.6, p.1495-1503, 2008.

BUENO, R.C.O.F.; PARRA, J.R.P.; BUENO, A.F. Biological characteristics and thermal requirements of a Brazilian strain of the parasitoid Trichogramma pretiosum reared on eggs of Pseudoplusia includens and Anticarsia gemmatalis. Biological Control, v.51, n.3, p.355-361, 2009.

CAÑETE, C.L. Seletividade de inseticidas a espécies de Trichogramma (Hymenoptera: Trichogrammatidae). 2005. 106f. Dissertação (Mestrado em Zoologia) - Universidade Federal do Paraná, Curitiba, 2005.

CARMO, E.L.; BUENO, A.F.; BUENO, R.C.O.F.; VIEIRA, S.S.; GOBBI, A.L.; VASCO, F.R. Seletividade de diferentes agrotóxicos usados na cultura da soja ao parasitoide de ovos Telenomus remus. Ciência Rural, v.39, n.8, p.2293-2300, 2009.

CARVALHO, G.A.; PARRA, J.R.P.; BAPTISTA, G.C. Ação residual de alguns inseticidas pulverizados em plantas de tomateiro sobre duas linhagens de 
Trichogramma pretiosum Riley, 1879 (Hymenoptera: Trichogrammatidae) em casa-de-vegetação. Ciência e Agrotecnologia, v.23, p.770-775, 1999.

CÔNSOLI, F.L.; ROSSI, M.; PARRA, J. Developmental time and characteristics of the immature stages of Trichogramma galloi and Trichogramma pretiosum (Hymenoptera: Trichogrammatidae). Revista Brasileira de Entomologia, v.43, p.271-275, 1999.

DHADIALLA, T.S.; CARLSON, G.R.; LE, D.P. New inseticides with ecdysteroidal and juvenile hormone activity. Annual Review of Entomology, v.43, p.545-569, 1998.

DIEZ-RODRÍGUEZ, G.I.; OMOTO, C. Herança da resistência de Spodoptera frugiperda (J.E. Smith) (Lepidoptera: Noctuidae) a lambda-cialotrina. Neotropical Entomology, v.30, n.2, p.311-316, 2001.

HASSAN, S.A. Guidelines for testing the effects of pesticides on beneficials organisms: description of test methods. In: HASSAN, S.A. (Ed.). Guidelines for testing the effects of pesticides on beneficials organisms. OILB/SROP, 1992. p.18-39. 1992. (Bulletin OILB/SROP 1992/XV/3).

HOHMANN, C.L. Efeito de diferentes inseticidas sobre a emergência de Trichogramma pretiosum

(Hymenoptera: Trichogrammatidae). Anais da Sociedade Entomológica do Brasil, v.20, p.59-65, 1991.

HOHMANN, C.L. Efeito de alguns inseticidas sobre adultos de Trichogramma pretiosum Riley. Anais da Sociedade Entomológica do Brasil, v.22, p.563-567, 1993.

IBGE (Brasília, DF). Safra 2007/2008: levantamento março/ 2008. Disponível em: <http://www.ibge.gov.br> Acesso em: 26 abr., 2008.

INOUE, M.S.R.; PARRA, J.R.P. Efeito da temperatura no parasitismo de Trichogramma pretiosum (Riley) sobre ovos de Sitotroga cerealella (Oliv.). Scientia Agricola, v.55, p.222-226, 1998.

MANZONI, C.G.; GRÜTZMACHER, A.D.; GIOLO, F.P.; HÄRTER, W.R.; CASTILHOS, R.V.; PASCHOAL, M.D.F. Seletividade de agroquímicos utilizados na produção integrada de maçã aos parasitóides Trichogramma pretiosum Riley e Trichogramma atopovirilia Oatan \& Platner (Hymenoptera: Trichogrammatidae). Bioassay, v.2, p.1-11, 2007.

MATOS, M.M. Seletividade a Trichogramma atopovirilia Oatan \& Platner 1983 de agroquímicos utilizados na citricultura paulista para o controle do bicho-furão-dos-citros Gymnandrosoma aurantianum Lima 1927. 2007. 54p. Dissertação (Mestrado em Entomologia) - Escola Superior de Agricultura "Luiz de Queiroz", Universidade de São Paulo, Piracicaba, 2007.

NÖRNBERG, S.D.; GRÜTZMACHER, A.D.; GIOLO, F.P.; JÚNIOR, G.J.E.; LIMA, C.A.B., GRÜTZMACHER,
D.D. Seletividade de formulações de glyphosate aplicado nos estádios imaturos de Trichogramma pretiosum. Planta daninha, v. 26, p. 611-617, 2008.

PARRA, J.R.P.; ZUCCHI, R. A. Trichogramma in Brazil: feasibility of use after twenty years of research. Neotropical Entomology, v.33, p.271-281, 2004.

PARRA, J.R.P.; BOTELHO, P.S.M.; CORRÊA-FERREIRA, B.S.; BENTO, J.M.S. Controle Biológico uma visão inter e multidisciplinar. In: PARRA, J.R.P.; BOTELHO, P.S.M.; CORRÊA-FERREIA, B.S.; BENTO, J.M.S. (Ed.). Controle biológico no Brasil: parasitóides e predadores. São Paulo, Manole, 2002. p.125-142.

PRATISSOLI, D.; PARRA, J.R.P. Desenvolvimento e exigências térmicas de Trichogramma pretiosum (Riley), criados em duas traças do tomateiro. Pesquisa Agropecuária Brasileira, v.35, p.1281-1288, 2000.

PRATISSOLI, D.; THULLER, R.T.; PEREIRA, F.F.; REIS, E.F. Ação transovariana de lufenuron $(50 \mathrm{~g} / \mathrm{L})$ sobre adultos de Spodoptera frugiperda (J. E. Smith) (Lepidoptera: Noctuidae) e seu efeito sobre o parasitóide de ovos Trichogramma pretiosum Riley (Hymenoptera: Trichogrammatidae). Ciência e Agrotecnologia, v.28, p. 9-14, 2004

REYNOLDS, S.E. The cuticule, growth regulators and moulting in insects: the essential background to the action of acylurea insecticides. Pesticide Science, v.20, p.131-146, 1987.

ROCHA, L.C.D.; CARVALHO, G.A. Adaptação da metodologia padrão da IOBC para estudos de seletividade com Trichogramma pretiosum Riley, 1879 (Hymenoptera: Trichogrammatidae) em condições de laboratório. Acta Scientiarum Agronomy, v.26, p.315-320, 2004.

SÁ, L.A.N.; PARRA, J.R.P. Biology and parasitism of Trichogramma pretiosum (Riley) (Hym.; Pyralidae) on Ephestia kuehniella (Zeller) and Heliothis zea (Boddie) (Lep.: Noctuidae) eggs. Journal of Applied Entomology, v.118, p.38-43, 1994.

SANTOS, A.C.; BUENO, A.F.; BUENO, R.C.O.F. Seletividade de defensivos agrícolas aos inimigos naturais. In: PINTO, A.S.; NAVA, D. E.; ROSSI, M. M.; MALERBO-SOUZA, D. T. (Ed.). Controle biológico de pragas na prática. Piracicaba: CP2, 2006. p.221-227.

SAS INSTITUTE. SAS user's guide: statistics: version 8.2. 6.ed. Cary, 2001. 201p.

WILLIAMS, T.; VALLE, J.; VIÑUELA, E. Is the naturally derived insecticide spinosad $\AA$ compatible with insect natural enemies? Biocontrol Science and Technology, v.13, p.459-475, 2003.

Recebido em 14/10/08

Aceito em 19/5/10 